\title{
Periodic host absence can select for higher or lower parasite transmission rates
}

Femke van den Berg (femke.vandenberg@bbsrc.ac.uk)

Nicolas Bacaer (nicolas.bacaer@ird.fr)

Johan A.J. Metz (j.a.j.metz@biology.leidenuniv.nl)

Christian Lannou (christian.lannou@grignon.inra.fr)

Frank van den Bosch (frank.vandenbosch@rothamsted.ac.uk)

\section{Approved by}

Ulf Dieckmann

Program Leader, EEP

June 2011 



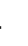

\section{Periodic host absence can select for higher or lower parasite transmission rates}

F. van den Berg ${ }^{1}$, N. Bacaer ${ }^{2}$, J.A.J. Metz ${ }^{3}$, C. Lannou ${ }^{4}$ and F. van den Bosch ${ }^{1}$

${ }^{1}$ Department of Biomathematics and Bioinformatics, Rothamsted Research, Harpenden, Hertfordshire AL5 2JQ, UK

${ }^{2}$ Institut de Recherche pour le Developpement, 32 avenue Henri Varagnat, Bondy 93143, France

${ }^{3}$ Mathematical Institute and Institute of Biology, Leiden University, P.O. Box 9512, 2300 RA Leiden, The Netherlands; Evolution and Ecology Program, International Institute of Applied Systems Analysis, A-2361 Laxenburg, Austria ${ }^{4}$ INRA, UMR 1290 Bioger, F-78850 Thiverval Grignon, France

Correspondence address: Dr. Femke van den Berg, Department of Biomathematics and Bioinformatics, Rothamsted Research, Harpenden, Hertfordshire AL5 2JQ, UK. Tel.: 01582763133 x2135, Fax: 01582-467907, E-mail: femke.vandenberg@bbsrc.ac.uk.

\section{Running title: Host absence and parasite evolution}

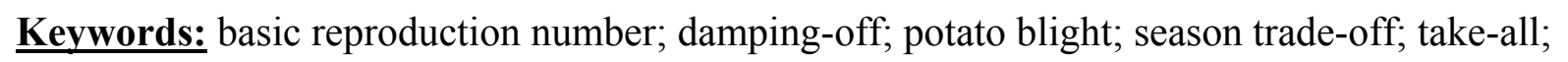
virulence trade-off; yellow rust

Word counts: TOTAL: 4310; Abstract: 193; Introduction: 1052; Materials and Methods: 869; Results: 928; Discussion: 1228; Acknowledgements: 40.

\section{References cited: 50}

Figures and tables: 1 figure; 2 tables

Online material: Appendix A (129 words) \& Appendix B (554 words) 


\section{Abstract}

2 This paper explores the effect of discontinuous periodic host absence on the evolution of

3 pathogen transmission rates by using $R_{0}$ maximisation techniques. The physiological

4 consequence of an increased transmission rate can be either an increased virulence, i.e. there

5 is a transmission-virulence trade-off or ii) a reduced between season survival, i.e. there is a

6 transmission-survival trade-off. The results reveal that the type of trade-off determines the

7 direction of selection, with relatively longer periods of host absence selecting for higher

8 transmission rates in the presence of a trade-off between transmission and virulence but lower

9 transmission rates in the presence of a trade-off between transmission and between season

10 survival. The fact that for the transmission-virulence trade-off both trade-off parameters

11 operate during host presence whereas for the transmission-survival trade-off one operates

12 during host presence (transmission) and the other (survival) during the period of host absence

13 is the main cause for this difference in selection direction. Moreover, the period of host

14 absence seems to be the key determinant of the pathogen's transmission rate. Comparing plant

15 patho-systems with contrasting biological features suggests that airborne plant pathogens

16 respond differently to longer periods of host absence than soil-borne plant pathogens. 


\section{Introduction}

2 Information on the evolutionary dynamics of pathogens has rapidly increased over the last

3 few decades for both directly transmitted pathogens (e.g. Anderson and May 1982, van

4 Baalen and Sabelis 1995, Dieckmann et al. 2002) and pathogens transmitting via distinct free-

5 living stages (Bonhoeffer et al. 1996, Day 2002b, Caraco and Wang 2008). Free-living stages

6 tend to have morphological and physiological adaptations that retard decay, allowing them to

7 survive outside the host for relatively long periods. On the other hand, plant pathogens like

8 rusts, potato blight and most biotrophic fungi affecting leaves disperse from host to host

9 through spores that deteriorate fast outside the host and have no way to survive by

10 themselves. Nevertheless, many of those pathogens relying on direct transmission are subject

11 to relatively large periods of host absence. This is particularly the case for pathogens of

12 cultivated crops, where harvest removes a large fraction of the host from the system.

13 Pathogens need then to bridge this period of host absence and they usually survive on

14 volunteer plants (seedlings resulting from accidental grain dispersal during harvest), crop

15 debris, seeds or infected tubers (Agrios 2005). These survival forms are fragile and transitory,

16 and lead to drastic reductions in the pathogen population size. The period of host absence is

17 then likely to put a strong selection pressure on the pathogen population.

19 Periodicity in host density has profound and well studied effects on the population dynamics

20 of human, animal and plant parasites (Altizer et al. 2006; Caswell 2001; Gubbins and Gilligan

21 1997a; Gubbins and Gilligan 1997b; Olinky et al. 2008), but few authors study the

22 evolutionary consequences of such host seasonality. Koella et al. (2005) showed that seasonal

23 forcing of a pathogen's sensitivity to environmental fluctuations can result in the selection of

24 reduced sensitivity to such fluctuations when environmental variability increases. Sorrell et al.

25 (2009) showed that host population variability achieved through seasonal forcing of the host 
1 birth rate can select for different levels of covert/silent infection depending on the forcing

2 amplitude. Although these models incorporate seasonality they assume that the host is present

3 throughout the season.

4

5 In this paper we focus on studying the direct effect of the length of a distinct period of host

6 absence between two consecutive host generations on the evolutionary dynamics of a directly

7 transmitted pathogen, in the presence of two qualitatively different trade-offs. We concentrate

8 on the evolution of transmission rate because this pathogen life-cycle parameter is closely

9 related to epidemic severity. Epidemic severity in its turn is closely related to host plant

10 fitness (in natural systems) and to crop yield loss (in agricultural systems). We firstly consider

11 a trade-off between parasite transmission and pathogen induced host death (i.e. virulence).

12 For directly transmitted pathogens, transmission between hosts increases with the rate of

13 inoculum production per generation. However, increased host resource exploitation increases

14 the host mortality rate and therewith decreases the average life expectancy of a lesion. This

trade-off will be referred to as the transmission-virulence trade-off. Although this trade-off is

16 one of the most readily accepted/studied trade-off within the area of evolutionary ecology

17 (Anderson and May 1982) its use has been criticised(Levin and Bull 1994). At the same time,

18 suggestions for the existence of such a trade-off are becoming increasingly available (see

19 Sacristan \& Garcia-Arenal (2008) for an overview)

21 Secondly, we consider a trade-off between parasite transmission and survival in the absence of the host. This could be, for example, a trade-off between pathogen transmission during host

23 presence and survival between host growth seasons on plant seeds/tubers, which in many

24 systems is a pathogen strategy to survive a period of crop host absence (Bull 1994, Agrios

25 2005, Montarry et al. 2007). The literature overview as provided in van den Bosch et al.

26 (2010) suggests that there is sufficient proof for the existence of this trade-off. Another 
1 example would be a trade-off between parasitic transmission success and saprotrophic

2 survival on host debris in soil-borne pathogens, with its existence recently proven for take-all

3 (Gaeumannomyces graminis) of wheat (Abang et al. 2006; Bailey et al. in prep.). This type of

4 trade-off will be referred to as the transmission-survival trade-off.

6 The simplest SIR model of epidemic dynamics, not including periodic host absence, is

$$
\begin{aligned}
& \frac{d S}{d t}=f(S, I)-d S-\beta S I \\
& \frac{d I}{d t}=\beta S I-\alpha I-d I
\end{aligned}
$$

8 Here, $S$ is the susceptible host density, $I$ is the infected host density, $f(S, I)$ is the host growth

9 rate representing the increase in either plant numbers or biomass (and thus receptive surface / tissue), $d$ is the natural host mortality rate, $\beta$ is the parasite transmission rate, $\alpha$ is the

11 pathogen induced host death rate, whereby all parameters are non-negative. The spores considered here are more or less randomly dispersed among plants. Moreover, they deteriorate quickly outside the host, so that transmission will occur before the infected host tissue dies. Transmission can thus be modelled by a mass action term, i.e. $\beta S I$. The diseasefree steady state of the system, $S^{*}$, is such that $f\left(S^{*}, 0\right)-d S^{*}=0$. The basic reproduction number of the pathogen, $R_{0}$, for this system is defined as the average number of new infections produced by a single newly infected individual in an otherwise disease free population, leading to,

$$
R_{0}=\frac{\beta S^{*}}{\alpha+d}
$$

It has been shown (e.g. van Baalen and Sabelis 1995 in the wake of Anderson \& May (1981)) that for model system (1) and a trade-off between transmission and virulence (our transmission-virulence trade-off) evolution will select for the transmission rate that maximises $R_{0}$. 
1 In this paper we will extend model equations (1) to include host seasonality. The basic

2 reproduction number, $R_{0}$, for this seasonal model will be calculated using methods developed

3 by e.g. Bacaer (2007) and Bacaer \& Guernaoui (2006). We will show that in our model,

4 including seasonal host absence, evolution still operates to maximise $R_{0}$. The framework thus

5 developed, in combination with the two trade-offs, is then used to answer the following

6 questions: Does a shorter host growing season (and thus a long period of host absence), select

7 for higher or lower transmission rates? Is host seasonality a key determinant of the

8 transmission rate that will be selected for or are other parameters in the system more

9 important? Are the effects of host seasonality on the transmission rate that is selected for

10 comparable across plant based patho-systems? We study these questions using a generic

11 qualitative analysis of the evolutionarily stable states as well as a system specific quantitative

12 analysis for four plant pathogen species.

\section{Model development}

14 Model equations: Model equations (1) are extended by including discontinuous host

15 presence, whereby the host is only present for a fixed period of time during a crop cycle. Let

$16 T$ be the length of one cycle and $\tau$ the period of this cycle in which the host is present $(0<\tau$

$17<$ ). The model is then given by

18

$$
\begin{aligned}
& \left.\begin{array}{l}
\frac{d S}{d t}=f(S, I)-d S-\beta S I \\
\frac{d I}{d t}=\beta S I-\alpha I-d I \\
P=0
\end{array}\right\} \text { if } n T^{+} \leq t \leq n T+\tau^{-} \\
& S=0 \\
& I=0 \\
& \frac{d P}{d t}=-\mu P
\end{aligned}
$$


1 At the start of a period of host presence $\left(n T^{+} \leq t \leq n T+\tau^{-}\right)$, we have $S\left(n T^{+}\right)+I\left(n T^{+}\right)=S_{o}$,

2 with $0<S_{0} \leq K$, i.e. a fixed density of crop is planted at the beginning of the season, of which

3 a certain fraction becomes infected, depending on the amount of pathogen that survived and

4 whereby $K$ is the carrying capacity of the host population. For $n T+\tau^{+} \leq t \leq(n+1) T^{-}$, we

5 have $S=I=0$, i.e. the crop is harvested at time $n T+\tau$. In the absence of the host the

6 pathogen switches to a survival strategy, $P$, whereby

$$
P\left(n T+\tau^{+}\right)=\theta_{1} I\left(n T+\tau^{-}\right) \text {for } 0<\theta_{1} \leq 1
$$

i.e. a fraction of the pathogen population has been removed during harvest. The remaining survival stages are subject to a constant death rate, $\mu$. At the beginning of each season the survival stages switch back to growing on the host resulting in

$$
I\left(n T^{+}\right)=\theta_{2} P\left(n T^{-}\right) \text {for } 0<\theta_{2} \leq 1
$$

i.e. a fraction of the pathogen population has been removed during planting and $P\left(n T^{+}\right)=0$. It is assumed that the newly infected crop is always infected with the pathogen at the beginning of the season. Note that $I$ and $P$ can be of the same type (e.g. infected host density and infected stubble density left on the field after harvest) or of different types (e.g. infected host density and infected seed density left on the field after harvest). See Figure 1a for an illustration of the model dynamics. In accordance with the pattern seen in Figure 1a we assume throughout that with the proceeding of time the solutions of (3) become T-periodic.

The trade-off relationships: The transmission-virulence trade-off, $\alpha(\beta)$. When a parasite increases its transmission rate, $\beta$, it also exploits its host's resources quicker which results in a larger pathogen induced host mortality, $\alpha$. This trade-off is the 'virulence' trade-off used in many evolutionary ecology studies (e.g. Anderson and May 1981, van Baalen and Sabelis 1995, Koella and Doebeli 1999). Note that for this trade-off both life-cycle parameters involved, transmission and disease induced host death, operate during the host growth season. 
1 We assume that increased transmission rates will become increasingly costly, and thus the

2 following properties hold:

$$
\frac{d \alpha}{d \beta}>0 \quad \text { and } \quad \frac{d^{2} \alpha}{d \beta^{2}}>0
$$

4 These are properties often used for this trade-off relationship (Anderson and May 1981, van

5 Baalen and Sabelis 1995, Koella and Doebeli 1999).

7 The transmission-survival trade-off, $\mu(\beta)$. For fungal plant pathogens spore production rates

8 and thus pathogen transmission are closely related to the pathogen's ability to colonize host

9 tissue and thus its mycelium growth rate. This has been found for pathogens with very different biological features, like Puccinia triticina, a pathogen of wheat (Pariaud et al. 2009a) and Leptosphaeria maculans, a pathogen of oil seed rape (Lô-Pelzer et al. 2009). High mycelium growth rates are also known to damage crop seeds (Cunfer and Johnson 1981b), reducing the probability of germination (Hewett 1975b, Cunfer and Johnson 1981b). For crop pathogens that survive on plant seeds between crop growing seasons this thus leads to a transmission-survival trade-off. A second group of examples for this trade-off is found in soilborne plant pathogens. When a soil-borne pathogen increases its transmission rate during the host growth season, $\beta$, it leaves fewer resources to invest in between-season survival, resulting in a higher between-season parasite death rate, $\mu$ (e.g. Abang et al. (2006); Bailey et al. in prep.). Note that the two life-cycle parameters of this trade-off operate during different parts of the season: transmission during the crop growth season and inoculum survival during the period of host absence. As for the transmission-virulence trade-off we assume that an increased transmission rate becomes increasingly costly and thus the following trade-off properties hold:

$$
\frac{d \mu}{d \beta}>0 \text { and } \quad \frac{d^{2} \mu}{d \beta^{2}}>0 .
$$


1 With these properties defined, the main conclusions of this study can be derived. However,

2 for the application of the results to specific pathogen systems we use $\alpha=\gamma \beta^{2}$ and $\mu=\eta \beta^{2}$.

$4 \quad$ Elasticity analysis: The elasticity, $e_{p}$, of the evolutionarily stable transmission rate, $\beta_{E S S}$, to

5 changes in a parameter is calculated as the proportional increase in $\beta_{E S S}$ in response to a

6 proportional increase in the parameter (Caswell, 2001)

$$
e_{p}=\frac{p}{\beta_{E S S}} \frac{d \beta_{E S S}}{d p}
$$

9 Case studies: We consider four fungal plant patho-systems for our quantitative analysis.

10 Puccinia striiformis, causing yellow rust on wheat; Phytophthora infestans, causing potato

11 late blight; Gaeumannomyces graminis var. tritici (Ggt), causing take all on wheat; and

Rhizoctonia solani, causing damping off on a wide range of hosts including cabbage,

13 cauliflower and radish. $P$. striiformis and $P$. infestans cause lesions on host leaves that

14 produce spores which are aerially transmitted (Agrios 2005). During the period of host

15 absence $P$. striiformis survives on volunteer plants (i.e. wheat plants escaped to the field margins) and P. infestans on tubers (Agrios 2005). G. graminis and R. solani are typical soil

17 borne pathogens, infecting the roots of their host plant (Agrios 2005). These pathogens

18 survive the period of host absence in the soil on infected roots or other soil organic material

19 (Hornby 1998, Herr 1976).

21 For each system the elasticities are studied in the presence of both the transmission-virulence and the transmission-survival trade-off. 


\section{Results}

2 The basic reproduction number, $\boldsymbol{R}_{0}$ : The dynamics of host in absence of disease is given by

$3 \quad S^{*}\left(n T^{+}\right)=S_{0}$ and $d S^{*}(t) / d t=f\left(S^{*}(t), 0\right)-d S^{*}$ if $n T^{+} \leq t \leq n T+\tau^{-} ; S^{*}(t)=0$ if

$4 n T+\tau^{+} \leq t \leq(n+1) T^{-}$and $I(t)=P(t)=0$ throughout the year. To derive $R_{0}$ the system is

5 linearised near this disease-free state, resulting in

6

$$
\begin{aligned}
& \frac{d I}{d t} \simeq \sigma_{1}(t) I-\omega_{1}(t) I \quad \text { for all } t \neq n T \\
& \frac{d P}{d t} \simeq \sigma_{2}(t) P-\omega_{2}(t) P \text { for all } t \neq n T+\tau
\end{aligned}
$$

and

$$
\begin{aligned}
& P\left(n T+\tau^{+}\right)=\theta_{1} I\left(n T+\tau^{-}\right) \\
& I\left(n T^{+}\right)=\theta_{2} P\left(n T^{-}\right)
\end{aligned}
$$

7 and where $\sigma_{1}(\mathrm{t}), \sigma_{2}(\mathrm{t}), \beta_{1}(\mathrm{t})$ and $\beta_{2}(\mathrm{t})$ are non-negative T-periodic functions given by

8

$$
\begin{aligned}
& \left.\begin{array}{l}
\sigma_{1}(t)=\beta S^{*}(t) \\
\omega_{1}(t)=\alpha+d
\end{array}\right\} \text { for } n T<t \leq n T+\tau \\
& \left.\begin{array}{l}
\sigma_{2}(t)=0 \\
\omega_{2}(t)=\mu
\end{array}\right\} \text { for } n T+\tau<t \leq(n+1) T .
\end{aligned}
$$

9 This "impulsive" system of differential equations can be rewritten for $n T^{-}<t<(n+1) T^{-}$as a single ordinary differential equation for $Z=I+P$

$$
\frac{d Z}{d t} \simeq A(t) Z-B(t) Z
$$

where $B(t)=b(t)-\log \left(\theta_{2}\right) \delta(t-n T)-\log \left(\theta_{1}\right) \delta(t-n T-\tau)$ and, e.g., $\delta(t-n T)$ is Dirac's delta and $A(t)=\sigma_{2}(t)$ for $n T+\tau^{+}<t<(n+1) T^{-}$

16 Bacaer and Guernaoui (2006) showed that for a system such as (9), 


$$
R_{0}=\frac{\int_{0}^{T} A(t) d t}{\int_{0}^{T} B(t) d t} .
$$

2 And we conclude that for our model equations (8)

3

4 where,

$$
R_{0 i}\left(\bar{S}_{r e s}\right)=\frac{\beta_{i} \tau}{(\alpha+d) \tau+\mu_{i}(T-\tau)-\log \left(\theta_{1}\right)-\log \left(\theta_{2}\right)} \cdot \bar{S}_{r e s}
$$

13 for the transmission-survival trade-off, where $\bar{S}_{\text {res }}$ denotes the mean host density in the

14 presence of the resident pathogen population and averaged over the period of host presence.

15 The basic reproduction number of the invader is thus the product of two functions,

$16 R_{0 i}\left(\bar{S}_{\text {res }}\right)=g\left(\beta_{i}\right) h\left(\beta_{\text {res }}\right)$, where $g$ only depends on the invader strategy and the other, $h$, only

17 on the resident strategy. For such cases Metz et al. (2008) and Mylius \& Diekmann (1995)

18 show that the ESS value of $\beta$ is found from maximising the function $g\left(\beta_{i}\right)$. Comparing

19 equations (13) and (14) with the expression of $R_{0},(10)$, we conclude that for our model the

20 ESS value of the transmission rate $\beta$ is that value of $\beta$ that maximises $R_{0}$. For a different proof 
1 using the even stronger result that out of any mixture of pathogen strains eventually the strain

2 with the highest value of $R_{0}$ prevails, see Appendix A.

3

4 The effect of host growing season, $\tau$, on the evolutionarily stable transmission rate, $\beta_{\mathrm{ESS}}$

5 Calculating the evolutionarily stable transmission rate by maximising $R_{0}$ we find

$$
\beta_{E S S} \frac{d \alpha}{d \beta}\left(\beta_{E S S}\right)-\alpha\left(\beta_{E S S}\right)=d+\mu \frac{T-\tau}{\tau}-\frac{\log \left(\theta_{1} \theta_{2}\right)}{\tau}
$$

7 and

$$
\beta_{E S S} \frac{d \mu}{d \beta}\left(\beta_{E S S}\right)-\mu\left(\beta_{E S S}\right)=(\alpha+d) \frac{\tau}{T-\tau}-\frac{\log \left(\theta_{1} \theta_{2}\right)}{T-\tau}
$$

9 for the transmission-virulence trade-off and the transmission-survival trade-off, respectively.

10 Both equations have the general structure $G\left(\beta_{\mathrm{ESS}}\right)=N(\tau)$, with

$11 G\left(\beta_{E S S}\right)=\beta_{E S S} \frac{d \alpha}{d \beta}\left(\beta_{E S S}\right)-\alpha\left(\beta_{E S S}\right)$ and $G\left(\beta_{E S S}\right)=\beta_{E S S} \frac{d \mu}{d \beta}\left(\beta_{E S S}\right)-\mu\left(\beta_{E S S}\right)$ for $(15)$ and $(16)$,

12 respectively. Calculating $G^{\prime}\left(\beta_{E S S}\right)=\beta_{E S S} \frac{d^{2} \alpha}{d \beta^{2}}$ and $G^{\prime}\left(\beta_{E S S}\right)=\beta_{E S S} \frac{d^{2} \mu}{d \beta^{2}}$ from (15) and (16),

13 respectively, and referring to equations (4) and (5) shows that $G\left(\beta_{\mathrm{ESS}}\right)$ is an increasing

14 function of $\beta_{\mathrm{ESS}}$. For the transmission-virulence trade-off $N(\tau)=d+\mu \frac{T-\tau}{\tau}-\frac{\log \left(\theta_{1} \theta_{2}\right)}{\tau}$,

which is a decreasing function of $\tau$. We thus conclude that for the transmission-virulence trade-off, independent of the precise shape of the trade-off curve, shorter host growing $\underline{\text { seasons }}$ select for high transmission rates. For the transmission-survival trade-off $N(\tau)=(\alpha+d) \frac{\tau}{T-\tau}-\frac{\log \left(\theta_{1} \theta_{2}\right)}{T-\tau}$, which is an increasing function of the length of the host growth season, $\tau$. We conclude that for the transmission-survival trade-off, independent of the precise shape of the trade-off curve, shorter host growing seasons select for low

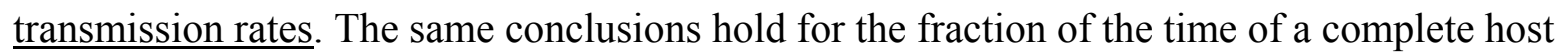
cycle over which the host is grown, $\tau / T$. See Appendix B for a graphical derivation of the 
1 results using the marginal value theorem in the manner in which this is done in foraging

2 theory (Charnov 1976, Stephens and Krebs 1986).

3

4 Elasticity analysis: Our results show (Table 2) that, for the transmission-virulence trade-off

5 and independent of the shape of the trade-off curve, the absolute value of the elasticity of the

6 evolutionarily stable transmission rate, $\beta_{\mathrm{ESS}}$, with respect to the length of the host growth

7 season is larger than the elasticity with respect to the parasite death rate in the period of host

8 absence. Whether or not the elasticity of $\beta_{\mathrm{ESS}}$ with respect to host growth season length is

9 larger or smaller than the elasticity with respect to the natural host death rate during the host

10 growth season depends on the parameter values.

12 For the transmission-survival trade-off the situation is even more clear-cut. The elasticity of

$13 \beta_{\text {ESS }}$ with respect to host growth season length is larger than the sum of the elasticities with respect to natural host death rate and virulence, as can be seen from

$$
\begin{aligned}
e_{\tau / T} & =\left(\frac{(\alpha+d) \tau}{T-\tau} \frac{T}{T-\tau}-\frac{\log \left(\theta_{1} \theta_{2}\right) \tau}{(T-\tau)^{2}}\right) \frac{1}{\beta_{E S S}^{2}}\left(\frac{\partial^{2} \alpha}{\partial \beta^{2}}\left(\beta_{E S S}\right)\right)^{-1} \\
& =\left(\left(\frac{\alpha \tau}{T-\tau}+\frac{d \tau}{T-\tau}\right) \frac{T}{T-\tau}-\frac{\log \left(\theta_{1} \theta_{2}\right) \tau}{(T-\tau)^{2}}\right) \frac{1}{\beta_{E S S}^{2}}\left(\frac{\partial^{2} \alpha}{\partial \beta^{2}}\left(\beta_{E S S}\right)\right)^{-1} \\
& =\left(e_{d}+e_{\alpha}\right) \frac{T}{T-\tau}-\frac{\log \left(\theta_{1} \theta_{2}\right) \tau}{(T-\tau)^{2}}>e_{d}+e_{\alpha}
\end{aligned}
$$

The case studies illustrate the trends in the elasticity of $\beta_{\mathrm{ESS}}$, whereby Table 1 gives the parameters according to which the system specific elasticities are calculated. All model parameters for the period of host absence can be estimated from literature sources available for the individual systems (see footnotes of Table 1). On the other hand for the period of host

21 absence the literature only provides estimates for the total survival success over this period, without making a distinction between the fractions of loss during harvest, $\theta_{1}$, and planting, $\theta_{2}$, 
1 and the between season pathogen mortality rate, $\mu$. Additional analysis has shown that when

2 the jumps in pathogen densities as a result of losses due to harvest and planting, i.e. $\theta_{1}$ and $\theta_{2}$

3 and the between season mortality rate, $\mu$, are amalgamated into a single parameter, $\mu^{\prime}$, such

4 that $\theta_{1} \theta_{2} e^{-\mu(T-\tau)}=e^{-\mu^{\prime}(T-\tau)}$ the qualitative results remain unchanged (results not shown). The

5 elasticities for the case in which the shape of the trade-off curves has been defined and thus

6 the calculations for the actual case studies are analysed for this amalgamated between season

7 mortality rate, $\mu$ ' (see Figure $1 \mathrm{~b}$ for an example of the dynamics). For the transmission-

8 survival trade-off the elasticity with respect to the host growth season length is at least twice

9 as large as the elasticity with respect to other parameters. The same holds for the

10 transmission-virulence trade-off for $P$. striiformis and P. infestans, the two aerially dispersed

11 pathogens. For the two soil borne pathogens, however, the elasticity with respect to the host

12 growth season length is smaller than the elasticity with respect to the host death rate.

\section{Discussion}

$14 \underline{\boldsymbol{R}}_{0}$ and discontinuous host growth: Periodic host absence is the rule in most plant-pathogen

systems. This is particularly true for agricultural systems, where planting and harvesting takes place at definite times and the regional scale. The behaviour of such systems during the crop growth period, which usually coincides with the epidemic phase of the pathogen cycle, has been studied extensively both experimentally and theoretically. In contrast, little information exists on the effect of the host absence period on pathogen dynamics and evolution. Here we proposed a simple and generic model, integrating host growth and host absence periods, to study the dynamic and evolutionary behaviour of pathogens in such seasonal systems. Our results show that for an SIR type epidemic model including periodic host absence the evolutionarily stable strategy can be calculated by $R_{0}$ maximisation, as also found in SIR models without periodic host absence. These results are based on a definition for $R_{0}$ in periodic environments as given in Bacaer and Guernaoui (2006) as well as a simple 
1 expression for $R_{0}$ derived by these authors for the case considered by us, plus the fact that thus

$2 R_{0}$ can be written as a product of a term depending on the parameters under evolution and a

3 term representing the effects of the resident dynamics. Despite the influence of the parameters

4 determining host density on the value of $R_{0}$, they do not affect the evolutionarily stable

5 parasite transmission rate (Equations (15) and (16)). Again this finding is in line with non-

6 seasonal systems (van Baalen and Sabelis 1995, Day 2002a, Metz et al. 2008).

7

8 The type of trade-off determines the direction of selection: Our results show that it depends on the type of trade-off relationship operating in the patho-system whether an increased period of host absence selects for a larger or a smaller transmission rate (Equations (14) \& (15) and Appendix B). For the transmission-virulence trade-off both parameters operate in the presence of the host, resulting in the selection of higher transmission rates. For the transmission-survival trade-off one component (transmission) operates during the host growing season and the other component (survival) during the period of host absence, resulting in the selection of lower transmission rates.

The transmission-virulence trade-off: Both trade-off components operate during the period of host presence and a pathogen will strive to maximise its inoculum build-up during the host growing season as this will increase its chances to survive the following period of host absence. An increased inoculum build-up can be achieved by an increased transmission rate but this goes paired with an increased virulence. With a decreasing host growing season the average life expectancy of a lesion decreases. A lesion with a short life expectancy has less need to spare the host, so on average, reproduction becomes relatively more important than host survival when the host growing season decreases, i.e., when the period of host absence increases. Thus, in the presence of the transmission-virulence trade-off shortening the host growing period selects for higher transmission rates. The 'traditional' models assuming 
1 continuous host presence predict that an increased base-line host mortality selects for

2 increased virulence (van Baalen and Sabelis 1995). As both a decreasing host growing season

3 and an increased host mortality rate result in a decrease in the average life expectancy of a

4 lesion it is not surprising that they have the same qualitative effect on the evolution of the

$5 \quad$ transmission rate and thus virulence.

7 The transmission-survival trade-off: The opposite dependency of the optimal transmission rate on the period of host presence for the transmission-survival trade-off is easier to understand. A reduced period of host growth means that the pathogen's ability to bridge the gap between two consecutive host growing seasons becomes proportionally more important.

11 The pathogen would thus strongly benefit from a reduced transmission rate during the host growing season as this leaves more resources to invest in the survival strategy and thus an increased chance to survive the longer period of host absence. In the presence of a transmission-survival trade-off shortening the host growing period will thus select for lower transmission rates.

These findings have consequences for the impact climate change might have on the dynamics of epidemics. Koelle et al. (2005) state that increased mean global air temperature due to climate change, likely leads to higher disease transmission and relaxation of the pathogen's overwintering restrictions. The combination of the two then increases disease severity. Climate change, however, also goes paired with faster crop maturation (Giménez 2006, Semenov 2009) and thus an increased period of host absence (since in our temperate climate crops can only be planted once a year). Our results imply that for patho-systems subject to a transmission-virulence trade-off only, climate change is likely to select for increased pathogen transmission. However, this does not necessarily result in an increased disease severity on a population level because the shorter host growing season leaves less time for the disease to 
1 develop. Our results also imply that in patho-systems subject to a transmission-survival trade-

2 off, where the period of host absence is the key determinant of the optimal transmission rate,

3 climate change is likely to select for lower transmission rates and therewith a reduced disease

4 severity.

The length of the period of host absence is a key determinant of pathogen transmission

7 rate: Our results show that the period of host absence is a key determinant of the evolutionarily stable transmission rate, with one marked exception: a patho-system facing a transmission-virulence trade-off when the parameter values are such that natural mortality exceeds the inter-crop pathogen death rate (Table 2).

Published data suggests that the transmission-virulence trade-off relationship operates in several aerially and vector dispersed plant pathogens (Montarry et al. 2006, Sacristan and Garcia-Arenal 2008, Pariaud et al. 2009b). The mode of survival between crop growing seasons of this group of pathogens also leads to the presence of a transmission-survival tradeoff. This holds for pathogens surviving on seeds or tubers (where mycelium growth rate correlates with spore production rate (transmission) as well as with damage done to the seed/tuber (Hewett 1975a, Cunfer and Johnson 1981a, Montarry et al. 2007)) and on volunteer/rogue plants (where large transmission rates exhaust the overwintering resource (Fisher et al. 2009)). Aerially dispersed plant pathogens thus seem to be subject to both tradeoffs. This implies that effects of environmental change and effects of disease control methods on the selection of transmission rate will depend on the balance between the opposite forces due to the two trade-offs. For these pathogens our results show that the length of the crop growing season/length of the period of host absence is by far the key determinant of the evolutionarily stable pathogen transmission rate. 
1 For the group of soil borne plant pathogens the existence of the transmission survival trade-

2 off is well established (Abang et al. 2006; Bailey et al. unpublished). We have, however, not

3 found any data suggesting the existence of a transmission-virulence trade-off for such

4 systems. This leads us to suspect that in soil borne plant pathogens the transmisison-survival

5 trade-off might be the key trade-off operating. This would imply that effects of environmental

6 change and effects of disease control methods on the evolutionarily stable transmission rate

7 are easy to predict. Any change such that the crop growth season shortens will select for

8 pathogens with a lower transmisison rate. As for the earially dispersed pathogens the length of

9 the crop growing season is the key determinant of the evolutionarily stable transisison rate.

\section{Acknowledgements}

11 Rothamsted Research receives support from the Biotechnology and Biological Sciences

12 Research Council (BBSRC) of the United Kingdom. F v d Berg was funded by an INRA-

13 BBSRC funded project entitled 'Epidemiological and evolutionary models for invasion and 14 persistence of disease'. 
Abang, M. M., M. Baum, S. Ceccarelli, S. Grando, C. C. Linde, A. Yahyaoui, J. Zhan, and B. A. McDonald. 2006. Differential selection on Rhynchosporium secalis during parasitic and saprophytic phases in the barley scald disease cycle. Phytopathology 96:12141222.

Agrios, G. N. 2005. Plant Pathology, 5th edition. Elsevier Academic Press, London.

Anderson, R. M., and R. M. May. 1981. The population dynamics of microparasites and their invertebrate hosts. Philos. T. Roy. Soc. B 291:451-524.

Anderson, R. M., and R. M. May. 1982. Coevolution of hosts and parasites. Parasitology 85:411-426.

Bacaer, N. 2007. Approximation of the basic reproduction number R-0 for vector-borne diseases with a periodic vector population. B. Math. Biol. 69:1067-1091.

Bacaer, N., and S. Guernaoui. 2006. The epidemic threshold of vector-borne diseases with seasonality. J. Math. Biol. 53:421-436.

Bonhoeffer, S., R. E. Lenski, and D. Ebert. 1996. The curse of the pharaoh: the evolution of virulence in pathogens with long living propagules. Proceedings of the Royal Society London - B Series 263:715-721.

Bull, J. J. 1994. Perspective: Virulence. Evolution 48:1423-1437.

Campbell, C. L., and L. V. Madden. 1990. Introduction to plant disease epidemiology. John Wiley \& sons, New Yorl.

Caraco, T., and I.-N. Wang. 2008. Free-living pathogens: life history constrains and strain competition. Journal of Theoretical Biology 250:569-579.

Charnov, E. L. 1976. Optimal foraging: the marginal value theorem. Theor. Popul. Biol. 9:129-136.

Chen, X. M. 2005. Epidemiology and control of stripe rust [Puccinia striiformis f. sp. tritici] on wheat. Can. J. Plant Pathol. 27:314-337.

Cook, A. R., W. Otten, G. Marion, G. J. Gibson, and C. A. Gilligan. 2007. Estimation of multiple transmission rates for epidemics in heterogeneous populations. PNAS 104:20392-20397.

Cunfer, B. M., and J. W. Johnson. 1981a. Trans. Brit. Mycol. Soc. 76:205.

Cunfer, B. M., and J. W. Johnson. 1981b. Relationship of glume blotch symptoms on wheat heads to seed infection by Septoria nodorum. T. Brit. Mycol. Soc. 76:205-211.

Day, T. 2002a. On the evolution of virulence and the relationship between various measures of mortality. P. Roy. Soc. B-Biol. Sci. 269:1317-1323.

Day, T. 2002b. Virulence evolution via host exploitation and toxin production in sporeproducing pathogens. Ecology Letters 5:471-476.

Dieckmann, U., J. A. J. Metz, M. W. Sabelis, and K. Sigmund. 2002. Adaptive dynamics of infectious diseases: in pursuit of virulence management. Cambridge University Press, Cambridge.

Fisher, A. J., B. J. Aegerter, T. R. Gordon, L. Smith, and D. M. Woods. 2009. Puccinia jaceae var. solstitialis teliospore priming on yellow starthistle. Phytopathology 99:6772.

Gibbs, R. J., and J. B. Reid. 1992. Comparison between net and gross root production by winter-wheat and by perennial ryegrass. New Zeal. J. Crop Hort. 20:483-487.

Gibson, G. J., A. Kleczkowski, and C. A. Gilligan. 2004. Bayesian analysis of botanical epidemics using stochastic compartmental models. PNAS 101:12120-12124.

Giménez, A. 2006. Climate change and variability in the mixed crop/livestock production systems of the Argentinean, Brazilian and Uruguayan Pampas. Instituto Nacional de Investigación Agropecuaria, Montevideo,Uruguay. 
Grasman, J., and G. van Straten. 1994. Predictability and nonlinear modelling in natural sciences and economics. Kluwer Academic publishers, Dordrecht.

Harris, P. M. 1992. The potato crop. The scientific basis for improvement. Chapman \& Hall, London.

Herr, L. J. 1976. I field survival of Rhizoctonia solani in soil and in diseased sugarbeets. Can. J. Microbiol. 22:983-988.

Hewett, P. D. 1975a. Trans. Brit. Mycol. Soc. 65:7.

Hewett, P. D. 1975b. Septoria nodorum on seedlings and stubble of winter wheat. T. Brit. Mycol. Soc. 65:7-18.

HGCA. 2008. The wheat growth guide.

Kadish, D., and Y. Cohen. 1989. Population dynamics of metalaxyl-sensitive and metalaxylresistant isolates of Phytophthora infestans in untreated crops of potato. Plant Pathol. 38:271-276.

Koella, J. C., and M. Doebeli. 1999. Population dynamics and the evolution of virulence in epidemiological models with discrete host generations. J. Theor. Biol. 198:461-475.

Koelle, K., M. Pascual, and M. Yunus. 2005. Pathogen adaptation to seasonal forcing and climate change. P. Roy. Soc. B-Biol. Sci. 272:971-977.

Levin, B. R., and J. J. Bull. 1994. Short-sighted evolution and the virulence of pathogenic microorganisms. Trends in Microbiology 2:76-81.

Lô-Pelzer, E., J. N. Aubertot, L. Bousset, X. Pinochet, and M. H. Jeuffroy. 2009. Phoma stem canker (Leptosphaeria maculans/L. biglobosa) of oilseed rape (Brassica napus): is the $\mathrm{G}_{2}$ Disease Index a good indicator of the distribution of observed canker severities? Eur. J. Plant Pathol.

Luo, Y., and S. M. Zeng. 1995. Simulation studies on epidemics of wheat stripe rust (Puccinia striiformis) on slow-rusting cultivars and analysis of effects of resistance components. Plant Pathol. 44:340-349.

Maillette, L. 1986. Canopy development, leaf demography and growth dynamics of wheat and three weed species growing in pure and mixed stands. J. Appl. Ecol. 23:939-944.

Metz, J. A. J., S. D. Mylius, and O. Diekmann. 2008. When does evolution optimize? Evol. Ecol. Res. 10:629-654.

Montarry, J., R. Corbiere, and D. Andrivon. 2007. Is there a trade-off between aggressiveness and overwinter survival in Phytophthora infestans? Funct. Ecol. 21:603-610.

Montarry, J., R. Corbiere, S. Lesueur, I. Glais, and D. Andrivon. 2006. Does selection by resistant hosts trigger local adaptation in plant-pathogen systems? J. Evolution. Biol. 19:522-531.

Mylius, S. D., and O. Diekmann. 1995. On evolutionary stable life histories, optimization and the need to be specific about density dependence. OIKOS 74:218-224.

Nowak, M. A., and K. Sigmund. 2002. Super- and coinfection: the two extremes. Pages 124137 in U. Dieckmann, J. A. J. Metz, M. W. Sabelis, and K. Sigmund, editors. Adaptive dynamics of infectious diseases. In pursuit of virulence management. Cambridge University Press, Cambridge.

Pariaud, B., C. Robert, H. Goyeau, and C. Lannou. 2009a. Aggressiveness components and adaptation to a host cultivar in wheat leaf rust. Phytopathology 99:869-878.

Pariaud, B., F. van den Berg, S. J. Powers, F. van den Bosch, and C. Lannou. 2009b. Is there a trade-off between latent period and spore production capacity in Puccinia triticina? Phytopathology Submitted.

Sacristan, S., and F. Garcia-Arenal. 2008. The evolution of virulence and pathogenicity in plant pathogen populations. Mol. Plant Pathol. 9:369-384.

Sanders, D. C. 1998. Radish. in. North Carolina Cooperative Extension Service Raleigh, North Carolina.

Semenov, M. A. 2009. Impacts of climate change on wheat in England and Wales. J. R. Soc. Interface 6:343-350. 
Sorrell, I., A. White, A. B. Pedersen, R. S. Hails, and M. Boots. 2009. The evolution of

2

12 covert, silent infection as a parasite strategy. P. Roy. Soc. B-Biol. Sci. 276:2217-2226.

Stephens, D. W., and J. R. Krebs. 1986. Foraging theory. Princeton University Press, Princeton, N. J.

van Baalen, M., and M. W. Sabelis. 1995. The dynamics of multiple infection and the evolution of virulence. The American Naturalist 146:881-910.

van den Bosch, F., B. Fraaije, F. van den Berg, and M. W. Shaw. 2010. Evolutionary bistability in pathogen transmission mode. P. Roy. Soc. B-Biol. Sci. In press.

Zadoks, J. C. 1961. Yellow rust on wheat studies in epidemiology and physiologic specialization. Tijdsch. Plantenz. 67:69-256. 


\section{Appendix A: Proof for $\boldsymbol{R}_{\mathbf{0}}$ maximisation}

2 The proof is an adaptation of the one given, e.g., by Nowak \& Sigmund (2002) for the case

3 without seasonality. Given that $S(t)=0$ for $n T+\tau<t<(n+1) T$, the original nonlinear

4 model can also be written as (cf. equations (7)-(9) in main text)

$$
\frac{d Z_{r}}{d t}=\beta_{r} Z_{r} S-B_{r}(t) Z_{r}, \quad \frac{d Z_{i}}{d t}=\beta_{i} Z_{i} S-B_{i}(t) Z_{i}
$$

Here subscripts ' $r$ ' and ' $i$ ' represent the resident and invader strain, respectively. Some

8 leads to

$$
\frac{1}{\beta_{r} Z_{r}} \frac{d Z_{r}}{d t}-\frac{1}{\beta_{i} Z_{i}} \frac{d Z_{i}}{d t}=-\frac{B_{r}(t)}{\beta_{r}}+\frac{B_{i}(t)}{\beta_{i}} .
$$

9 Assume $R_{0}^{(i)}>R_{0}^{(r)}>1$. Integrating and letting $t$ tend to infinity, we find

$$
\begin{aligned}
\frac{1}{\beta_{r}} \log \left(\frac{Z_{r}(t)}{Z_{r}(0)}\right)-\frac{1}{\beta_{i}} \log \left(\frac{Z_{i}(t)}{Z_{i}(0)}\right) & =-\frac{1}{\beta_{r}} \int_{0}^{t} B_{r}(u) d u+\frac{1}{\beta_{i}} \int_{0}^{t} B_{i}(u) d u \\
& \sim \frac{t}{T}\left(-\frac{1}{\beta_{r}} \int_{0}^{T} B_{r}(u) d u+\frac{1}{\beta_{i}} \int_{0}^{T} B_{i}(u) d u\right), \\
& \sim \frac{t}{T}\left(-\frac{1}{R_{0}^{(r)}}+\frac{1}{R_{0}^{(i)}}\right) \int_{0}^{\tau} S^{*}(u) d u, \\
& \rightarrow-\infty .
\end{aligned}
$$

11 So either $Z_{i}(t) \rightarrow+\infty$ or $Z_{r}(t) \rightarrow 0$ as $t \rightarrow \infty$. The crop population size is constrained by a carrying capacity which means that $Z_{i}(t) \rightarrow+\infty$ is impossible, resulting in $Z_{r}(t) \rightarrow 0$ being

13 the only possible solution, leading to "competitive exclusion". The only strain remaining is 14 the one with the highest value of $R_{0}$. 


\section{Appendix B: Marginal value theory and the evolution of}

\section{2 pathogen transmission rates}

3 In this appendix we show that the $R_{0}$ maximisation problem as described in this paper is

4 susceptible to the marginal value theorem and graphical analysis from evolutionary ecology

5 (Charnov 1976, Stephens and Krebs 1986). To improve comparison with the existing

6 literature we assume that not the transmission rate, but the virulence (in the case of the

7 transmission-virulence trade-off) or the between season pathogen death rate (in the case of the

8 transmission-survival trade-off) evolves. The trade-off parameters are however subject to the

9 same constraint, which means that this redefinition does not affect the results as discussed in

10 the main text.

11 The transmission-virulence trade-off: The optimal virulence, given the trade-off constraints

$$
\beta=g(\alpha), \frac{d g}{d \alpha}>0 \text { and } \frac{d^{2} g}{d \alpha^{2}}<0
$$

can be calculated from $d R_{0} / d \alpha=0$, leading to

$$
g(\alpha)=\frac{d g}{d \alpha}\left[\alpha+d-\mu+\mu T / \tau-\log \left(\theta_{1} \theta_{2}\right) / \tau\right]
$$

In analogy to the graphic solutions from optimal foraging theory (Charnov 1976, Stephens and Krebs 1986) this expression can be depicted as illustrated in Figure B1a. The curve represents the trade-off following the constraints as set out in (B1) and the straight line represents the solution of (B2) and is the tangent of trade-off curve. The optimal strategy is given by the point where these two lines intersect. From this graph it can immediately be seen that $\left(d+\mu \frac{T-\tau}{\tau}-\frac{\log \left(\theta_{1} \theta_{2}\right)}{\tau}\right)$ and hence the ESS virulence value, $\alpha_{E S S}$, increases when the host growing period, $\tau$, decreases. 


$$
\frac{d[\tau / T] g(\alpha)}{d[\tau / T] \alpha}=\frac{[\tau / T] g(\alpha)}{[\tau / T](\alpha+d)+[(T-\tau) / T] \mu-\log \left(\theta_{1} \theta_{2}\right)}
$$

2 which states that the increment in year averaged infectivity per increment in year averaged

3 death rate should match the year averaged infectivity divided by the year averaged death rate.

4 From this it can directly be derived that decreasing the host growing season, $\tau$, means that the

5 relative contribution of virulence, $\alpha$, to the year averaged death rate decreases, which allows

6 for the selection of higher virulence and therewith higher transmission rates.

7 The transmission-survival trade-off: The optimal between season pathogen death rate,

8 given the trade-off constraints

$$
\beta=g(\mu), \frac{d g}{d \mu}>0 \text { and } \frac{d^{2} g}{d \mu^{2}}<0
$$

can be calculated from $d R_{0} / d \mu=0$, leading to

$$
g(\mu)=\frac{d g}{d \mu}\left[(\alpha+d) \frac{\tau}{T-\tau}+\mu-\frac{\log \left(\theta_{1} \theta_{2}\right)}{T-\tau}\right]
$$

12 Figure B1b shows how the optimal between season pathogen death rate can be derived

13 graphically in analogy to the graphic solutions from optimal foraging theory (Charnov 1976,

14 Stephens and Krebs 1986). From this graph it can immediately be seen that

$15 \frac{(\alpha+d) \tau}{T-\tau}-\frac{\log \left(\theta_{1} \theta_{2}\right)}{T-\tau}$ and hence the ESS between season pathogen death value, $\mu_{E S S}$,

16 decreases when the host growing period, $\tau$, decreases. The ESS condition can also be written in a marginal value form

$$
\frac{d[\tau / T] g(\mu)}{d[\tau / T] \mu}=\frac{[\tau / T] g(\mu)}{[\tau / T](\alpha+d)+[(T-\tau) / T] \mu-\log \left(\theta_{1} \theta_{2}\right)}
$$

which states that the increment in year averaged infectivity per increment in year averaged death rate should match the year averaged infectivity divided by the year averaged death rate.

21 From this it can directly be derived that decreasing the host growing season, $\tau$, means that the relative contribution of the between season pathogen death rate, $\mu$, to the year averaged death 
1 rate increases, which suggests the pathogen should invest in a better survival strategy leading

2 to the selection of lower between season pathogen death rates and therewith lower

3 transmission rates.

4
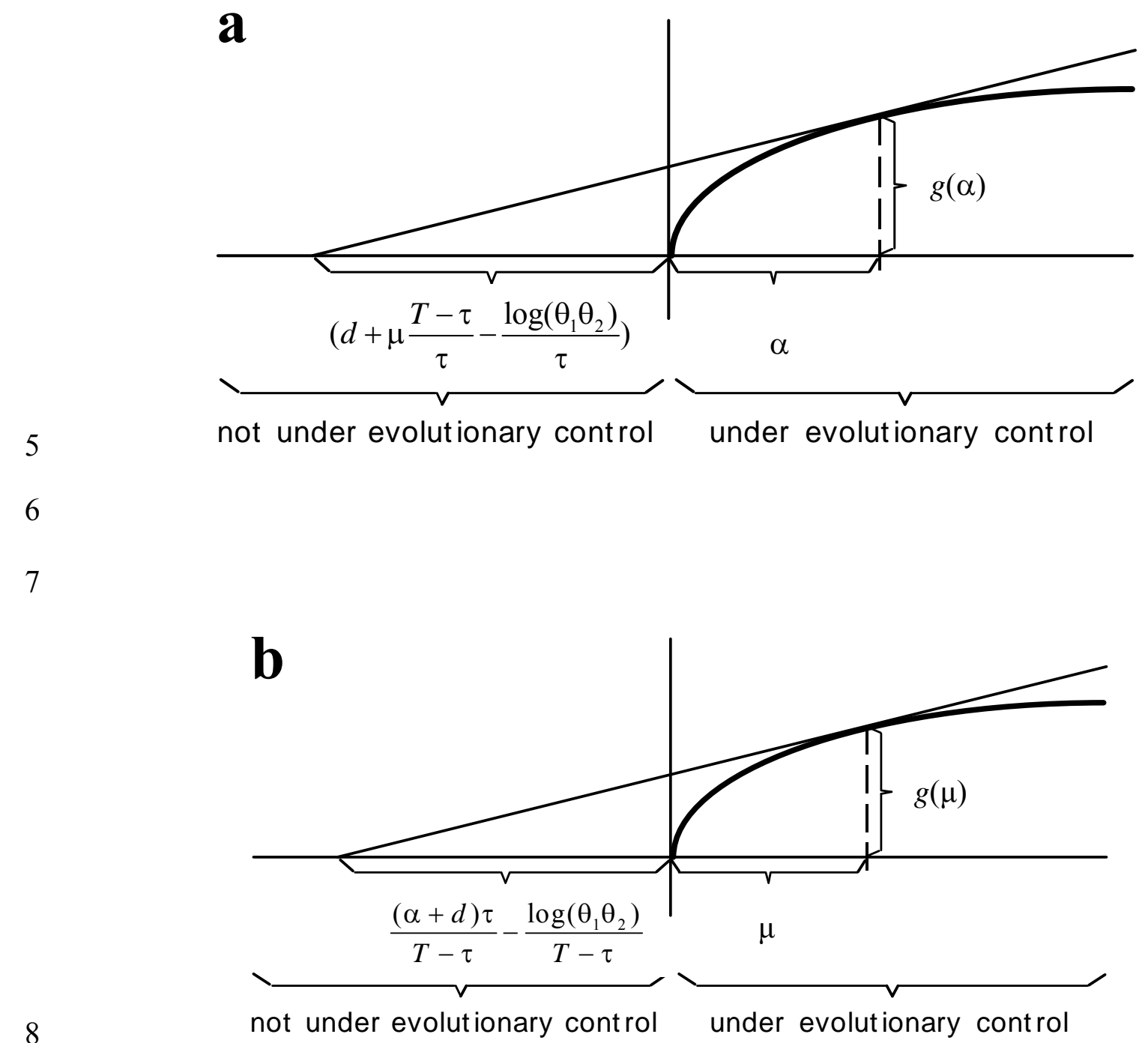

10 Figure B1. Graphical representation of the optimal strategy for a hypothetical trade-off

11 relation between a) transmission and virulence and b) transmission and between season

12 pathogen death rate. The tangent is given by the solution of $d R_{0} / d \alpha=0$ and $d R_{0} / d \mu=0$ for

13 the transmission-virulence and the transmission-survival trade-off, respectively. The optimal

14 strategy is given by the point where the two lines intersect. 


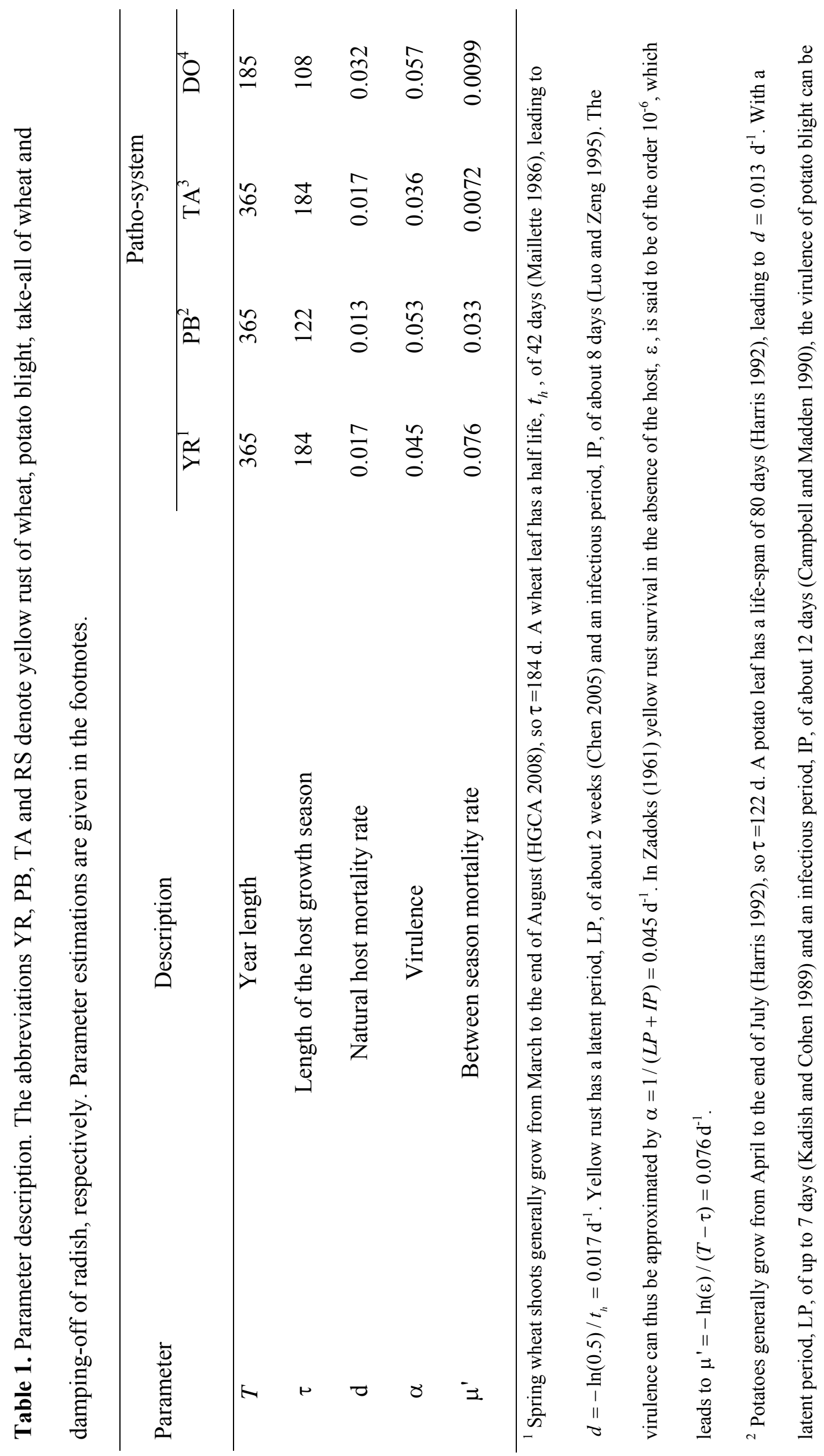




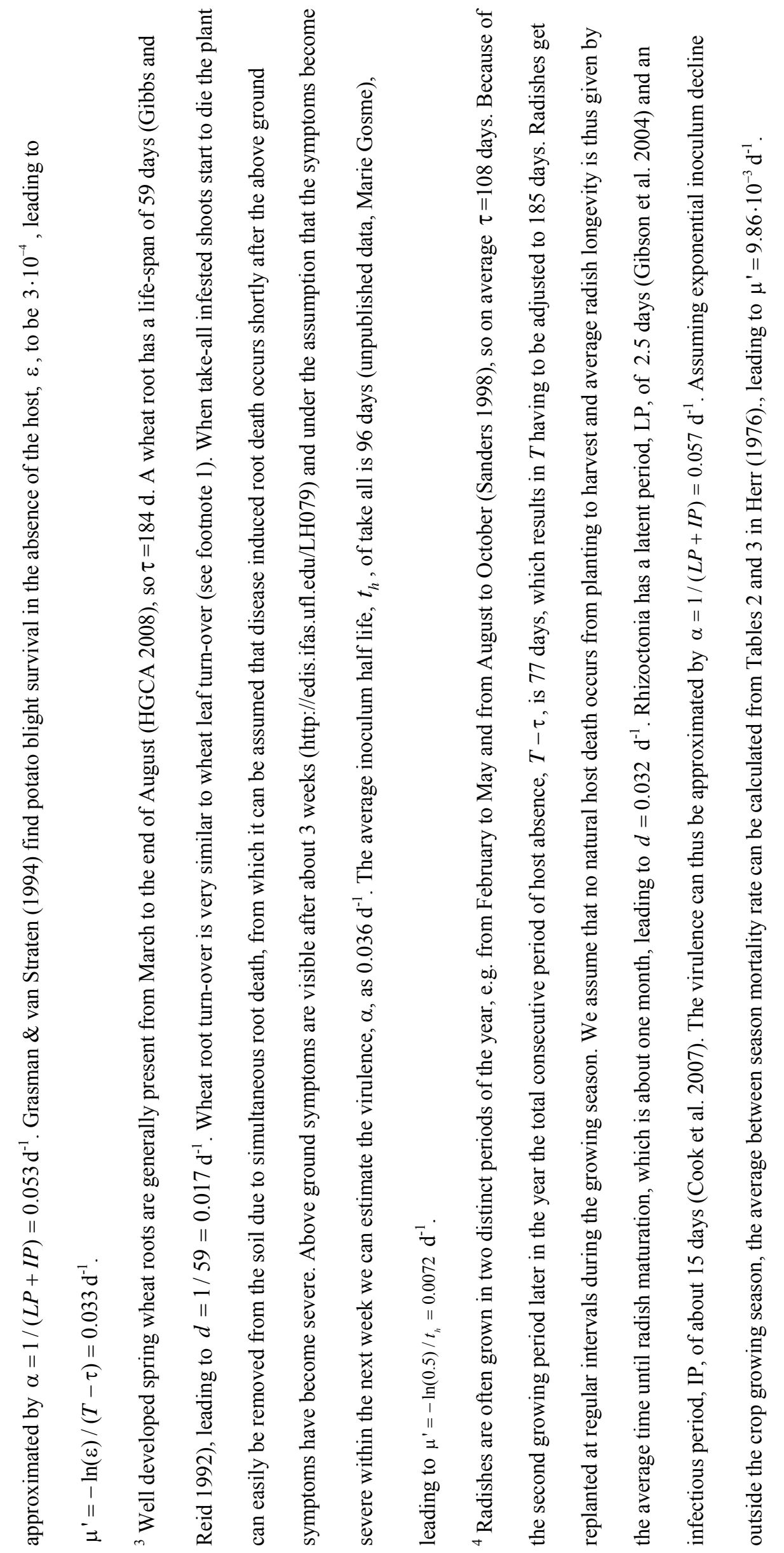




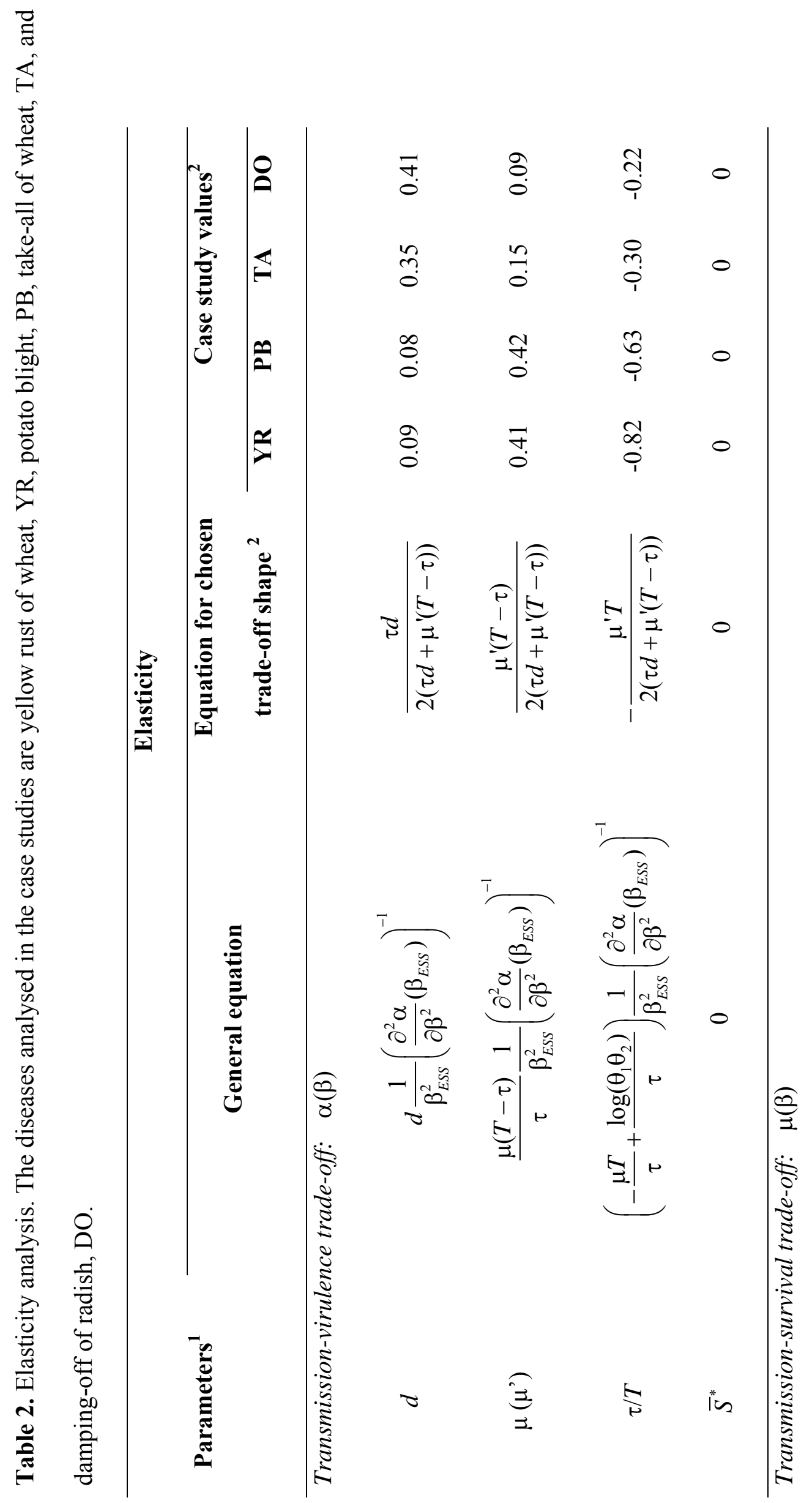




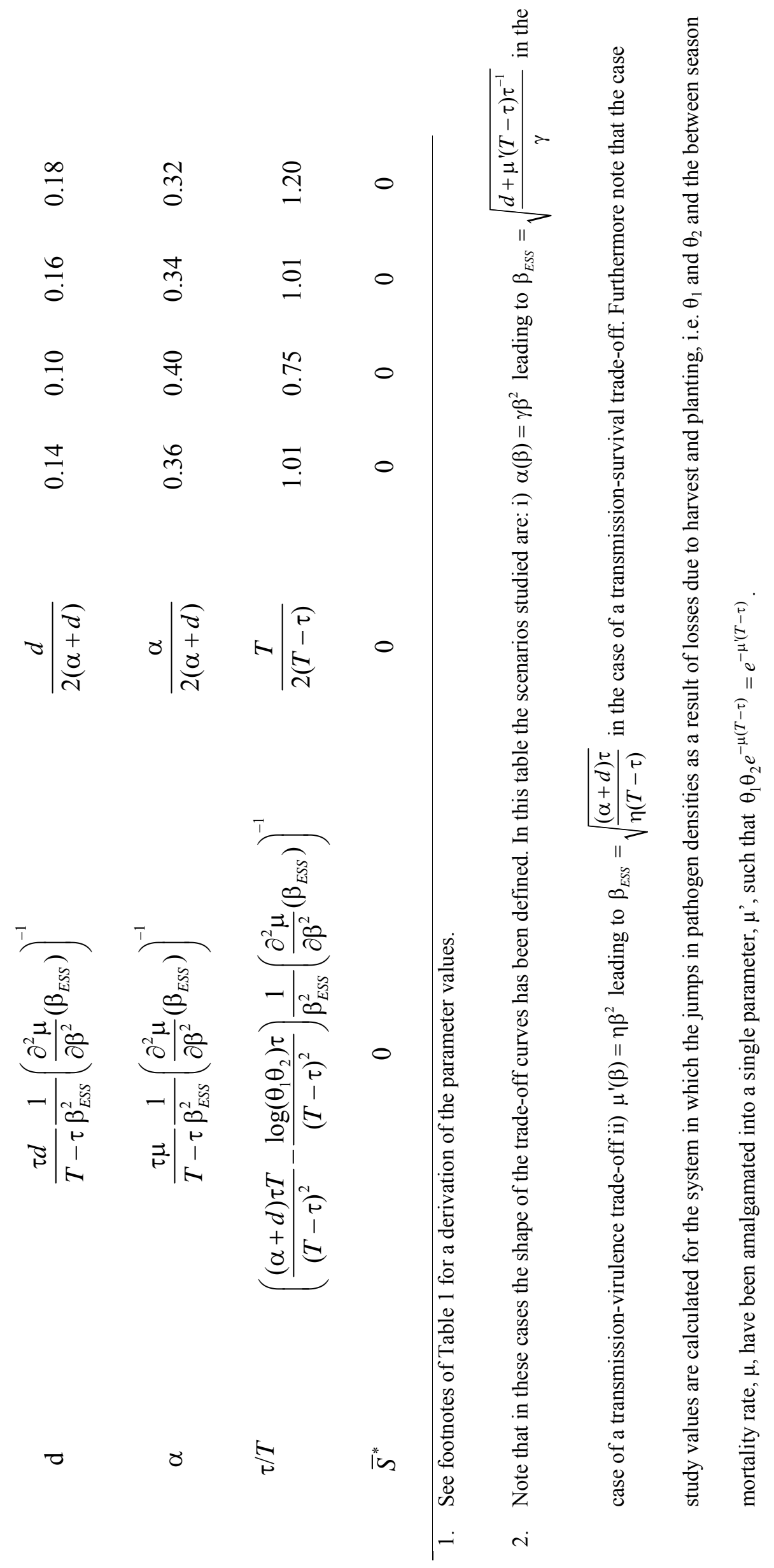


Figure 1. System dynamics. (a) Illustration of the dynamics as described by equations (3). (b) Example of the healthy host (grey) and infected host (black) density dynamics in the presence of a transmission-virulence trade-off for take-all disease entering a wheat population after the first complete host cycle. Here the jumps in pathogen density have been amalgamated into the exponential decline parameter such that $\theta_{1} \theta_{2} e^{-\mu(T-\tau)}=e^{-\mu^{\prime}(T-\tau)}$. The disease parameters are based on take-all of wheat, whereas the other parameters are chosen such as to maximise illustrative clarity, leading to $\tau=$ $184 ; d=0.017 ; \mu^{\prime}=0.0072 ; f(S, I)=r S\left(1-\frac{S+I}{K}\right)$ with $r=0.07$ and $\mathrm{K}=15 ; \alpha(\beta)=$ $\gamma \beta^{2}$ with $\gamma=562.5, \beta=0.0065$ and $\alpha=0.024$ and $S_{0}=3$.

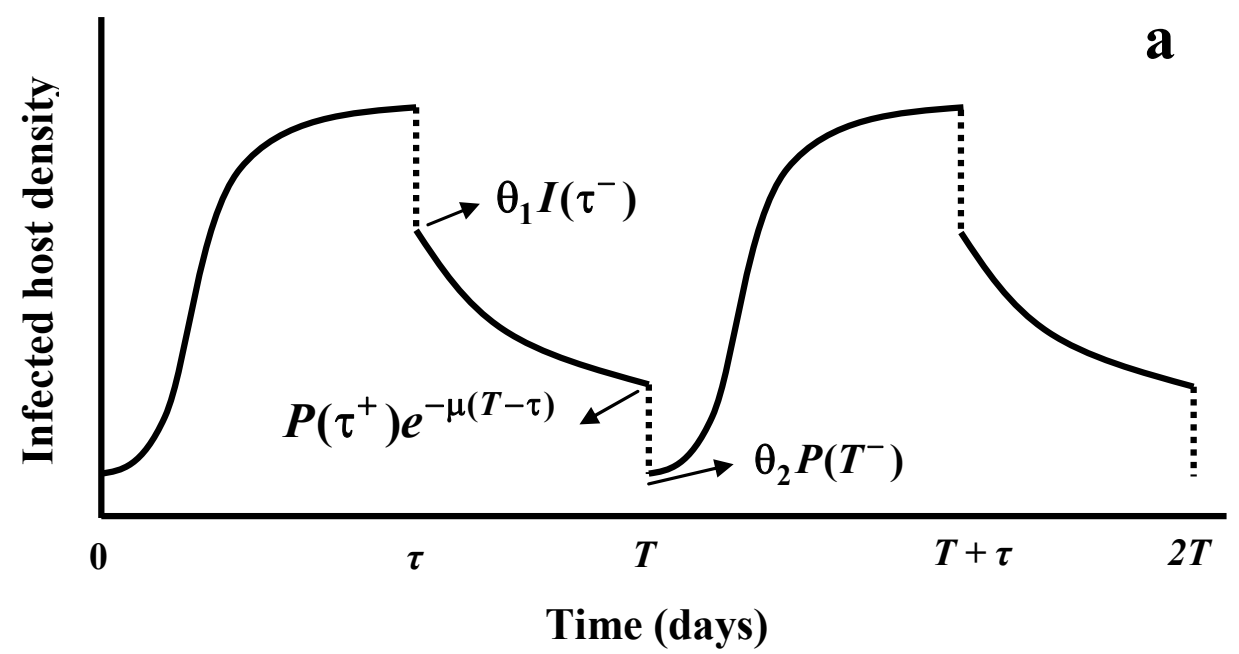

b 


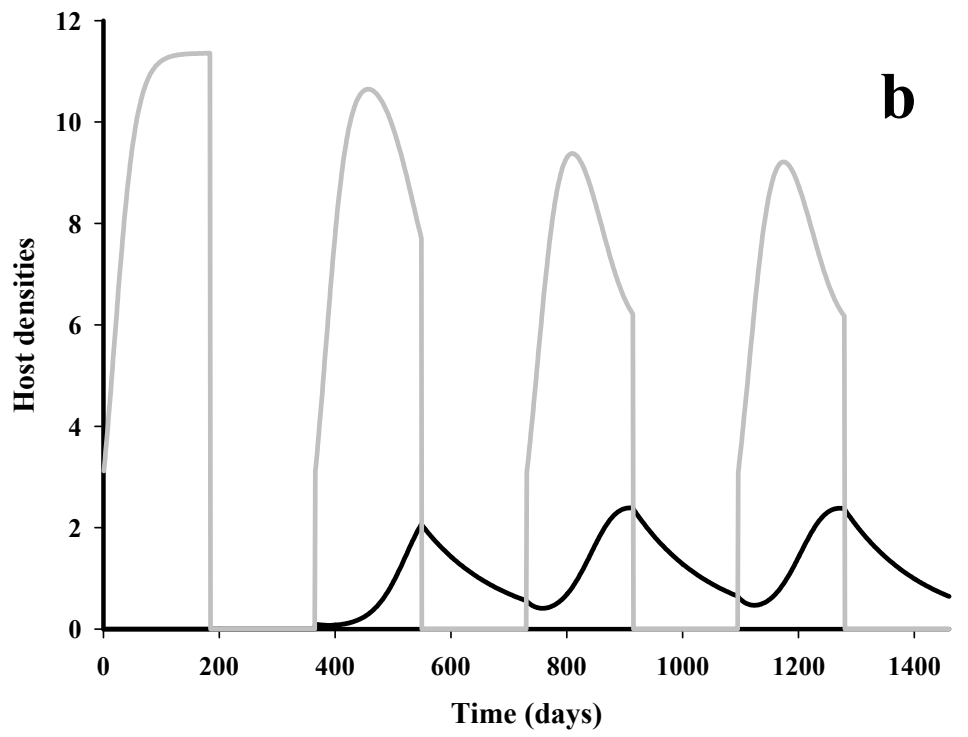

